\title{
THEOREMS ON COMPACT TOTALLY DISCONNECTED SEMIGROUPS AND LATTICES ${ }^{1}$
}

\section{KATSUMI NUMAKURA}

In the theory of topological algebras, it is well-known that a compact totally disconnected group (or a compact totally disconnected ring) is a projective limit of finite groups (or finite rings). In $\$ 2$ of this paper we shall establish a theorem on compact totally disconnected Hausdorff semigroups which is similar to that for compact totally disconnected groups.

The theorem can be applied to other compact totally disconnected algebraic systems. For example, it can be applied to compact totally disconnected rings, compact totally disconnected distributive lattices, etc.

In $\S 3$, we shall prove an imbedding theorem for compact totally disconnected distributive lattices which is well-known in the discrete case $[1] .^{2}$

Throughout the paper we will use the terminology of Wallace $[3 ; 4]$ and [5]. Thus a mob $S$ is a Hausdorff space together with a continuous associative multiplication. $A \subset S$ is a $T$-ideal, where $T$ is a nonvacuous subset of $S$, if and only if $T A \subset A$ and $A T \subset A$. Moreover, a topological lattice $L$ is a Hausdorff space together with a pair of continuous functions $\wedge: L \times L \rightarrow L, \vee: L \times L \rightarrow L$ satisfying the usual conditions for lattice operations.

The author wishes to acknowledge the advice and helpful suggestions of Professor A. D. Wallace in the preparation of this paper.

1. Let $S$ be a topological space and $\Delta$ the diagonal of the product space $S \times S: \Delta=\{(x, x) ; x \in S\}$.

Let $\Re$ be a subset of $S \times S$.

(1) $\Re$ is called reflexive if $\Re \supset \Delta$.

Let us denote by $\sigma$ a map from $S \times S$ into itself defined by $\sigma(x, y)$ $=(y, x)$ for $(x, y) \in S \times S$. Then $\sigma$ is a homeomorphism from $S \times S$ onto itself and $\sigma^{2}$ is the identical map.

(2) $\Re$ is called symmetric if $\sigma(\Re)=\Re$.

Let $\Re_{1}, \Re_{2}$ be two subsets of $S \times S$. We define a subset $\Re_{1} \circ \Re_{2}$ of $S \times S$ as follows: $(x, y) \in \Re_{1} \circ \Re_{2}$ if and only if there exists an element $z$ in $S$ with $(x, z) \in \Re_{1}$ and $(z, y) \in \Re_{2}$.

(3) $\Re$ is called transitive if $\Re \circ \Re \subset \Re$.

Received by the editors August 10, 1956 and, in revised form, October 3, 1956.

1 This work was supported by the National Science Foundation, U.S.A.

2 Numbers in brackets refer to the bibliography at the end of this paper. 
If $\Re$ is a subset of $S \times S$ which is reflexive, symmetric and transitive, then we can define an equivalence relation with respect to $\Re$ in $S$ by

$$
x \equiv y \bmod \Re \text { if and only if }(x, y) \in \Re .
$$

Let us denote by $S / \Re$ the set of equivalence classes with respect to $\Re$. Define the natural map $f: S \rightarrow S / \Re$ by $f(x)=$ "the equivalence class $\bmod \Re$ containing $x$." Topologize $S / \Re$ as usual, i.e. a subset $V^{*}$ of $S / \Re$ is open if and only if $f^{-1}\left(V^{*}\right)$ is open in $S$, so $S / \Re$ is a topological space and $f$ is a continuous map from $S$ onto $S / \Re$.

Now we can easily get the following:

Lemma 1. Let $S$ be a compact Hausdorff space and $\Re$ an open subset of $S \times S$ which is reflexive, symmetric and transitive. Then $S / \Re$ is a finite discrete space.

Lemma 2. Let $S$ be a compact, totally disconnected Hausdorff space and $\mathfrak{D}$ an open subset of $S \times S$ containing the diagonal $\Delta$. Then there exists, in $\mathfrak{D}$, an open subset $\Re$ of $S \times S$ which is reflexive, symmetric and transitive.

Proof. Since $S$ is a compact totally disconnected Hausdorff space, we can find a finite number of open and closed subsets $V_{1}, \cdots, V_{k}$ in $S$ such that

$$
\Delta \subset \bigcup_{i=1}^{k}\left(V_{i} \times V_{i}\right) \subset \mathfrak{O}
$$

Let $U_{1}=V_{1}$ and $U_{i}=V_{i} \backslash\left(V_{1} \cup \ldots \cup V_{i-1}\right)$ for $i=2,3, \cdots, k$. Then $U_{1}, \cdots, U_{k}$ are open subsets of $S$ with $U_{i} \cap U_{j}=\square$ for $i \neq j$ and $\Delta \subset \bigcup_{i=1}^{k}\left(U_{i} \times U_{i}\right) \subset \mathcal{D}$. Let $\Re=\bigcup_{i=1}^{k}\left(U_{i} \times U_{i}\right)$, then $\Re$ is the open subset of $S \times S$ which we wanted.

2. We are going to consider a compact mob $S$ instead of a space $S$. The product space $S \times S$ is also a compact mob with the componentwise product.

Lemma 3. Let $S$ be a compact mob and $\mathfrak{A}$ an open submob of $S \times S$ which is reflexive, symmetric and transitive. Then $S / \mathfrak{A}$ is a finite, discrete mob.

Proof. By Lemma $1, S / \mathfrak{A}$ is finite and discrete. Let $a^{*}, b^{*}$ be two elements of $S / \mathfrak{A}$ and let $a^{*}=f(a), b^{*}=f(b)$, where $f$ is the natural map from $S$ onto $S / \mathscr{Y}$ and $a, b$ are elements of $S$. Define the product of $a^{*}$ and $b^{*}$ by $a^{*} b^{*}=f(a b)$. Then this product is uniquely defined. In fact, if $a^{*}=f\left(a^{\prime}\right)$ and $b^{*}=f\left(b^{\prime}\right), a^{\prime}, b^{\prime} \in S$, then $\left(a, a^{\prime}\right) \in \mathfrak{A}$ and $\left(b, b^{\prime}\right)$ 
$\in \mathfrak{A}$. Since $\mathfrak{A}$ is a submob of $S \times S$, we get $\left(a b, a^{\prime} b^{\prime}\right) \in \mathfrak{A}$, whence $f(a b)=f\left(a^{\prime} b^{\prime}\right)$. Now, associativity of the multiplication can easily be proved. Thus $S / \mathfrak{A}$ is a finite, discrete mob.

In this case, we call the natural map $f$ the natural homomorphism.

Lemma 4. Let $S$ be a compact totally disconnected mob and $\mathfrak{O}$ an open subset of $S \times S$ containing the diagonal $\Delta$. Then there exists, in $\mathfrak{D}$, an open submob $\mathfrak{A}$ of $S \times S$ which is reflexive, symmetric and transitive.

Proof. Let $\Re$ be an open, reflexive, symmetric and transitive subset of $S \times S$ in $\mathfrak{D}$ (see Lemma 2). We denote by $\mathfrak{A}$ the union of all $\Delta$-ideals contained in $\Re$, that is the largest $\Delta$-ideal in $\Re$. Then, by the same proof as that of Lemma 1 of [2], $\mathfrak{A}$ is open. $\Delta$ itself is a $\Delta$-ideal contained in $\Re$, so $\Delta \subset \mathfrak{A}$, i.e. $\mathfrak{A}$ is reflexive.

Since $\Re$ is symmetric, $\sigma(\mathfrak{A}) \subset \Re$ and $\sigma(\mathfrak{A})$ is also a $\Delta$-ideal. Therefore $\sigma(\mathfrak{A}) \subset \mathfrak{A}$, and $\sigma(\mathfrak{A})$ must be equal to $\mathfrak{A}$, that is, $\mathfrak{A}$ is symmetric.

To prove the transitivity of $\mathfrak{A}$, pick an element $(x, y)$ from $\mathfrak{A} \circ \mathfrak{A}$. Then there exists an element $z$ in $S$ with $(x, z) \in \mathfrak{A} \subset \Re$ and $(z, y)$ $\in \mathfrak{H} \subset \Re$. So $(x, y) \in \Re \circ \Re \subset \Re$, because $\Re$ is transitive. Hence $\mathfrak{A} \circ \mathfrak{A} \subset \mathfrak{R}$. On the other hand it can readily be seen that $\mathfrak{A} \circ \mathfrak{A}$ is a $\Delta$-ideal. Thus $\mathfrak{A} \circ \mathfrak{A} \subset \mathfrak{A}$, namely $\mathfrak{A}$ is transitive.

Now we shall show that $\mathfrak{A}$ is a submob of $S \times S$. Let $(x, y)$ and $\left(x^{\prime}, y^{\prime}\right)$ be two elements of $\mathfrak{A}$. Then, since $\mathfrak{A}$ is a $\Delta$-ideal, we have $\left(x x^{\prime}, y x^{\prime}\right) \in \mathfrak{A}$ and $\left(y x^{\prime}, y y^{\prime}\right) \in \mathfrak{A}$. From the transitivity of $\mathfrak{A}$ we get $\left(x x^{\prime}, y y^{\prime}\right) \in \mathfrak{A}$. Hence $\mathfrak{A}$ is a submob of $S \times S$.

Theorem 1. A compact totally disconnected mob is a projective limit of finite discrete mobs.

Proof. Let $S$ be a compact totally" disconnected mob" and $\Delta$ the diagonal of $S \times S$. Then, by Lemma 4 , we can find a family $\mathfrak{F}=\left\{\mathfrak{A}_{\lambda}\right.$; $\lambda \in \Lambda$ \} of open, reflexive, symmetric and transitive submobs of $S \times S$ with $\left(1^{\circ}\right) \cap_{\lambda \in \Lambda} \mathfrak{A}_{\lambda}=\Delta$ and $\left(2^{\circ}\right)$ for any two members $\mathfrak{A}_{\lambda}, \mathfrak{A}_{\mu}$ of $\mathfrak{F}$ there exists a third member $\mathfrak{A}_{\nu}$ such that $\mathfrak{A}_{\nu} \subset \mathfrak{A}_{\lambda} \cap \mathfrak{A}_{\mu}$.

Let $S_{\lambda}=S / \mathscr{A}_{\lambda}$ then, by Lemma 3 , each $S_{\lambda}$ is a finite discrete mob.

For $\lambda, \mu \in \Lambda$, define $\lambda>\mu$ by $\mathfrak{A}_{\lambda} \subset \mathfrak{A}_{\mu}$, then $\Lambda$ becomes a directed set and it can readily be seen that for $\lambda>\mu$ there is an (open continuous) homomorphism $f_{\mu}^{\lambda}: S_{\lambda} \rightarrow S_{\mu}$ with $f_{\mu}^{\lambda} f_{\nu}^{\mu}=f_{\nu}^{\lambda}$ if $\lambda>\mu>\nu$. Thus $S$ is a projective limit of finite discrete mobs $S_{\lambda}$ 's.

3. Let $L$ be a topological lattice. Then $L$ is a mob with respect to the join operation and also with respect to the meet operation.

Lemma 5. If $L$ is a compact totally disconnected distributive lattice and $I_{0}$ is the lattice $\{0,1\}$ then for $x, y \in L$ and $x \neq y$ there is a continuous homomorphism $f: L \rightarrow I_{0}$ such that $f(x) \neq f(y)$. 
Proof. Since $x \neq y$ the element $(x, y)$ of the product space $L \times L$ is not contained in the diagonal $\Delta$. Then, by Lemma 4 , there exists an open, reflexive, symmetric and transitive submob $\mathfrak{A}$ with respect to the join operation.

Next, take the largest $\Delta$-ideal $\mathfrak{M}$ contained in $\mathfrak{A}$ (i.e. the union of all $\Delta$-ideals contained in $\mathfrak{U}$ ) with respect to the meet operation. Then, using the distributivity of $L$, we can easily prove that $\mathfrak{M}$ is an open, reflexive, symmetric and transitive subset of $L \times L$ which is closed under join and meet operations. Moreover, the element $(x, y)$ is not contained in $\mathfrak{M}$. Then, by a similar proof to that of Lemma 3 , we can conclude that $L / \mathfrak{M}$ is a finite discrete distributive lattice and there is the natural homomorphism $g: L \rightarrow L / \mathfrak{M}$ such that $g(x) \neq g(y)$.

Since $L / \mathfrak{M}$ is a finite discrete distributive lattice we can find a (continuous) homomorphism $h: L / \mathfrak{M} \rightarrow I_{0}$ such that $h g(x) \neq h g(y)$ (see $[1])$. Let $f=h g$. Then $f$ is a continuous homomorphism from $L$ onto $I_{0}$ which is just what we wanted.

Theorem 2. A compact totally disconnected distributive lattice can be imbedded (algebraically and topologically) in a compact Boolean lattice.

Proof. Let $L$ be a compact totally disconnected distributive lattice and $I_{0}$ the lattice $\{0,1\}$.

We denote by $H\left(L, I_{0}\right)$ the set of all continuous homomorphisms of $L$ into $I_{0}$ and by $B(L)$ the set of all functions from $H\left(L, I_{0}\right)$ to $I_{0}$. Then $B(L)$ is a compact Boolean lattice (the cartesian product of $I_{0}$ with itself one time for each element of $\left.H\left(L, I_{0}\right)\right)$.

Define a function $\phi: L \rightarrow B(L)$ by $\phi(x)(\alpha)=\alpha(x)$, where $x \in L$, $\alpha \in H\left(L, I_{0}\right)$. Then, using Lemma 5 , it can readily be seen that $\phi$ is a topological isomorphism of $L$ into $B(L)$. Thus $L$ can be imbedded in the compact Boolean lattice $B(L)$.

One may prove that a compact totally disconnected distributive lattice is a projective limit of finite discrete distributive lattices. This is an immediate consequence of Theorem 1 and the proof of Lemma 5.

\section{REFERENCES}

1. G. Birkhoff, Lattice theory, New York, 1948.

2. R. J. Koch and A. D. Wallace, Maximal ideals in compact semigroups, Duke Math. J. vol. 21 (1954) pp. 681-685.

3. A. D. Wallace, The structure of topological semigroups, Bull. Amer. Math. Soc. vol. 61 (1955) pp. 95-112.

4. - Retractions in semigroups (not yet published).

5. - The center of a compact Hausdorff lattice is totally disconnected (not yet published).

Tulane UNIVERSITY 